\title{
Pitfalls of statistical hypothesis testing: multiple testing
}

The word "five" is missing from the third sentence of the fifth paragraph in the online answers to this Endgames article by Philip Sedgwick (BMJ 2014;349:g5310, doi:10.1136/bmj. g5310). The sentence should have read: "For example, the probability of a significant result when there are two, three, four, and five hypothesis tests is $0.098,0.143,0.186$, and 0.226 , respectively."

Cite this as: BMJ 2014;349:95624

๑ BMJ Publishing Group Ltd 2014 